\title{
Les métamorphoses du livre
}

Les rendez-vous de lédition : le livre et le numérique

Roger Chartier

Bibliothèque

Ex-2 Centre

Pompitiou 


\section{Les métamorphoses du livre}

Les rendez-vous de l'édition : le livre et le numérique

\section{Roger Chartier}

DOI : 10.4000/books.bibpompidou.1699

Éditeur : Éditions de la Bibliothèque publique d'information Année d'édition : 2001

Date de mise en ligne : 13 janvier 2015

Collection : Paroles en réseau

ISBN électronique : 9782842462048

\section{Donerstition ebooks}

http://books.openedition.org

\section{Édition imprimée}

ISBN : 9782842460693

Nombre de pages : 35

\section{Référence électronique}

CHARTIER, Roger. Les métamorphoses du livre : Les rendez-vous de l'édition : le livre et le numérique. Nouvelle édition [en ligne]. Paris : Éditions de la Bibliothèque publique d'information, 2001 (généré le 02 février 2021). Disponible sur Internet : <http://books. openedition.org/bibpompidou/1699>. ISBN : 9782842462048. DOI : https://doi.org/10.4000/books.bibpompidou.1699. 


\section{Les métamorphoses du livre}

Les rendez-vous de l'édition : le livre et le numérique

Roger Chartier

Bibliothèque

E-Sentre

puthiese dintormation

Pompidou 


\title{
Les métamorphoses du livre
}

\author{
Conférence inaugurale du 8 janvier 2001
}

J'ai pensé qu'il y avait plusieurs manières d'entrer dans la question du livre et du numérique, qui est le thème des interventions de ce cycle de 2001. Je ne pourrai pas toutes les traiter, mais je voudrais toutes les évoquer, rapidement les deux premières et la troisième d'une façon plus approfondie, à partir de ma propre pratique de travail de recherche.

Ces trois manières d'aborder cette question - qui seront sans doute illustrées au cours du cycle -, sont la sociologie des pratiques de lecture, l'économie de l'édition, et I'histoire longue de la culture écrite, et particulièrement du livre. Trois modes différents, mais qu'on peut essayer de lier. Je vais donc vous les présenter.

Une première approche possible serait en termes de sociologie des pratiques de lecture: vous savez que ces dernières années des enquêtes se sont multipliées dans ce domaine du savoir. S'appuyant sur des séries statistiques, elles peu-

vent avoir été menées à une échelle nationale fournissant des données brutes et ouvertes au commentaire. $C^{\prime}$ 'est le cas avec les enquêtes que réalise tous les cinq ans le ministè re de la Culture et qui sont publiées sous le titre Pratiques culturelles des Français. Elles permettent de suivre les évolutions de la fréquentation du livre, et plus généralement d'autres imprimés. Les sociologues, eux, se sont concentrés sur des enquêtes qui portent sur des populations spécifiques de lecteurs: les étudiants un livre dirigé par Emmanuel Fraisse (Les étudiants et la lecture, PUF, 1993) -, les adolescents - un livre récent de Christian Baudelot, $M$ arie $C$ artier, et Christine D etrez (Et pourtant ils lisent, Seuil, 1999). On peut aussi penser à ces enquêtes sur les lecteurs en bibliothèque qu'a menées - avec un rôle pionnier - , le service des Etudes et recherche de la Bibliothèque publique d'information qui s'est ainsi donné un miroir d'elle-

Roger Chartier, historien, directeur d'études à l'École des hautes études en sciences sociales.

(C) Roger Chartier, 2001. 
même. Une troisième modalité d'enquête dans le domaine de la sociologie de la lecture consiste à suivre des trajectoires de lecteurs, et I'ouvrage de Gérard $M$ auger, Claude F. Poliak et Bernard Pudal (H istoires de lecteurs, $N$ athan, 1999) concerne un certain nombre de destins de lecteurs.

Dans cette perspective, avec ces enquêtes à différentes échelles - données nationales, populations particulières, individus singuliers - , il me semble que le monde du numérique tient encore peu de place, et que les enquêtes se sont attachées massivement à la description et à la quantification des pratiques de lecture face à leurs objets traditionnels, imprimés pour l'essentiel, depuis le livre jusqu'au journal, au magazine ou à la revue, etc. Lorsque le monde du numérique apparaît dans ces enquêtes, et d'une manière plus réflexive que statistique, c'est sous deux aspects. Comme concurrent du livre et de la lecture, avec l'analyse des diffé rents lieux de ces concurrences: le transfert des dépenses de la consommation culturelle vers les objets électroniques et vers d'autres consommations que celles liées à l'achat de l'imprimé et aux pratiques de lecture classique; ou en termes de concurrence sur le temps des loisirs, avec l'importance accordée au temps passé devant l'ordinateur, mais pour des usages qui ne sont pas caractérisés fondamentalement comme des lectures, mais qui peuvent être de l'ordre du ludique, de la distraction, du divertissement, prolongeant ainsi le temps passé devant d'autres écrans, ceux de la télévision ou du cinéma; ou encore, en termes de transfert ou de déplacement de répertoires - opposition classique entre un monde numérique d'images et un monde imprimé de textes, un monde du jeu et un monde du sérieux; ou encore un monde porteur des icônes contemporaines de la culture des mass media opposé à I'héritage canonique, classique, des répertoires textuels anciens abandonnés au profit du premier. II y a donc là une première perspective dans laquelle le monde du numérique est fondamentalement un monde d'images et un monde du divertissement et de la distraction, opposé à ce qu'était le monde de l'imprimé.

L'autre figure de cette présence du numérique dans la perspective d'une sociologie de la lecture est une figure inverse, à savoir l'idée qu'avec le monde du numérique 
existe un support pour une reconquête de la lecture, et donc une place retrouvée ou développée de l'écrit. Le constat qui fonderait cette seconde perspective est évidemment le constat lié à la nature spécifique des écrans du monde du numérique, qui rend obsolète l'ancienne problématique, si fameuse depuis M CLuhan, qui opposait un monde d'écrans - qui était un monde d'images, et d'images parlantes, et mobiles -, à une galaxie ancienne, celle de Gutenberg, qui était un monde de l'écrit, de l'imprimé, de la lecture. Les écrans du numérique sont des écrans qui portent, pas seulement, mais fondamentalement, de l'écrit. Et l'expression quel'on emploie souvent, « de l'écrit à l'écran », doit être entendue non pas comme la substitution des écrans au monde de l'écrit, mais la présence sur l'écran, affiché à l'écran, de l'écrit. Et c'est sans doute de là que vient ce lien assez paradoxal, dans nos sociétés, entre d'un côté une prolifération de l'écrit - et une prolifération qui est puissamment appuyée sur le monde de la communication numérique -, et de l'autre côté cette thématique obsédante, éplorée, mélancolique, de la mort du lecteur. Ce serait là un premier axe de réflexion. Je ne peux pas l'approfondir parce que ce n'est pas mon domaine de compétences, mais il me semble qu'on aurait cette ambivalence du numérique dans le monde de la sociologie, des pratiques de lecture, comme concurrent et comme support.

Si I'on pense à une autre dimension, qui serait celle de l'économie de l'édition, on voit ici que le point de départ est aussi un diagnostic d'inquiétude, plus particulièrement un diagnostic énoncé en termes de crise à l'intérieur de l'édition traditionnelle, et tout particulièrement dans certains secteurs. Celui qui m'est le plus proche - et qui est sans doute proche à beaucoup d'entre vous - , est le secteur des sciences humaines, des essais, des livres de savoir mais, me semblet-il, ce que je vais dire ne serait pas sans pertinence pour le secteur de la fiction. Ce diagnostic de crise s'énonce à travers des chiffres: si l'on suit les statistiques du Syndicat national de l'édition, la décennie quatre-vingt dix a été une décennie de recul, de recul du nombre global de volumes publiés - je parle ici pour la France bien sûr, et pour les sciences humaines et sociales, mais aussi dans un sens très large, pour l'en- 
semble de la littérature qu'on appellerait d'essais ou de savoir - , et un recul du nombre d'exemplaires vendus pour chaque titre. Et ce mouvement de recul des années quatre-vingt dix a été accompagné, paradoxalement, là encore, au moins jusqu'aux toutes dernières années, par l'accroissement du nombre de titres publiés, ce qui n'est pas le signe d'une vitalité confiante, mais d'une recherche de nouveaux marchés, de nouveaux publics, à travers de nouvelles formes pour compenser le recul du nombre d'exemplaires vendus, donc finalement publiés par titre, qui entraîne le recul global du nombre de volumes vendus. Voilà un diagnostic qui s'énonce donc en terme statistiques, qui s'énonce aussi en terme de discours, et l'on retrouverait ici une figure que les historiens connaissent bien, c'était une expression qui était chère à Ernest Labrousse, celle d'imputation au politique; cette situation un peu paradoxale dans laquelle une activité qui relève très globalement, très massivement de l'entreprise privée tourne ses griefs et ses demandes du côté de la puissance publique considérant qu'un élément clé, sinon unique de la crise, provient des faiblesses de
I'application ou du manque de la réglementation étatique. Tous les débats qui ont mobilisé ces dernières années les éditeurs, les bibliothécaires et les lecteurs tournent autour de cette imputation aux politiques, que ce soit le débat autour du prix unique du livre et de son extension au marché de la commande publique, ou le débat autour du droit de copie - du droit de photocopie -, ou plus récemment le débat autour du droit de prêt en bibliothèque. Et à partir de cette tension, qui fait considérer la réglementation étatique lorsqu'elle est défaillante comme une des raisons, sinon la raison principale, des difficultés, et qui la fait considérer corollairement, comme la manière de sauvegarder la bonne santé du secteur éditorial, on a vu se forger dans les discours un certain nombre de justifications capables d'assurer ces revendications, et des justifications en termes généraux: la protection des droits patrimoniaux des auteurs ou la légitimité du prix à payer pour accéder à la culture écrite, de la même façon qu'on paye un prix pour accéder à d'autres modalités culturelles.

Comme historien il serait intéressant - c'est juste une parenthèse - , d'observer ce mécanisme dans 
lequel des données objectives de crise sont transformées en des discours - des discours qui se tournent vers la puissance publique, mais qui pour se faire accepter de l'opinion publique doivent se fonder sur des principes généraux. II serait assez intéressant de comparer ce mécanisme avec ce qu'il a été en d'autres moments de crise de l'édition, par exemple à la fin du $x^{\prime} x^{e}$ siècle, lorsque cette idée même d'une crise de l'édition est née - liée, à ce moment-là, à une crise de surproduction par rapport à ce qu'était capable d'absorber le marché - , ou encore au XVIII siècle, en Angleterre et en France, lorsque l'ancien système des privilèges a été mis en question. Et I'on retrouverait non pas des contenus de discoursidentiques, mais des stratégies de discours et des systèmes de perception de la crise et de ses solutions possibles, qui pourraient être mis en parallèle.

$D$ ans ce deuxième contexte - celui d'une économie de l'édition fondée sur un diagnostic de crise, et débouchant sur cette imputation au politiques -, la référence au numérique joue un rôle, là encore, ambivalent. D'un côté, elle peut être présentée, pensée comme sinon la solution à la crise, du moins une façon de la soulager. C'est ainsi qu'on a proposé que pour des secteurs où l'accès à la publication imprimée n'est plus possible, parce que plus rentable, l'édition électronique pourrait prendre le relais. O n le voit dans deux domaines particuliers, du côté des livres spécialisés issus de recherches - des thèses, des livres d'érudition, des études monographiques, tout le secteur qu'aux États-Unis on qualifie de « monographs » - et pour lesquels des modalités d'édition électronique, pouvant être des formes d'édition à la demande, viendraient se substituer à ce qui était l'édition imprimée, puisque, comme l'a montré le grand historien américain Robert $D$ arnton, dans un article devenu fameux traduit dans la revue Le Débat, à partir du moment où les bibliothèques universitaires ne sont plus acheteuses de ces monographs, les presses universitaires elles-mêmes ne les publient plus, et des livres qui auraient eu accès, sans problème, à l'édition imprimée, ne le peuvent plus dans cette situation. De là, la proposition, déjà suivie par un certain nombre, de donner une forme d'édition électronique à ces ouvrages érudits, spécialisés, monographiques qui ne trouvent plus de publication imprimée. 
L'autre secteur, qui serait presque à l'opposé du secteur des monographs, concerne le secteur des ouvrages encyclopédiques, dans lequel le pas a déjà été franchi, puisque certaines des plus fameuses encyclopédies, I'Encyclopæalia Britannica, ou I'Encyclopzelia U niversalis sont uniquement proposées sous forme électronique. Et ici joue, bien sûr, la réduction du coût, mais aussi la facilité des mises à jours et la commodité de la consultation pour des ouvrages où la lecture n'est pas une lecture continue de la première à la dernière page, mais une lecture de recherche, de confrontation, de croisement.

On voit que cette figurelà du monde numérique, dans l'économie de l'édition, n'est pas séparée du contexte de l'édition classique, mais qu'elle est la recherche, pour un certain nombre d'ouvrages, soit par nécessité, soit par commodité, d'une forme nouvelle, à l'intérieur d'un monde éditorial qui reste pour d'autres ouvrages dominé par la forme imprimée. Et de là le fait que ces formes-là, en particulier la première, celle des ouvrages spécialisés, peuvent être prises en charge par des institutions ou des éditeurs tout à fait classiques.
Une seconde perspective du monde du numérique dans l'économie de l'édition est évidemment plus audacieuse, plus anticipatrice, peut-être plus utopique: elle se fonde sur l'idée qu'à terme le livre imprimé sera remplacé par le livre électronique, et la condition en est qu'une transformation déjà entamée, pas toujours perçue peut-être à sa juste mesure, a modifié les attentes, les compétences, les pratiques de lecture d'un certain nombre de communautés de lecteurs. Cette deuxième perspective, qui est une perspective non pas de cohabitation à l'intérieur d'un monde éditorial entre la forme imprimée classique et des secteurs numériques, maisl'idée d'une substitution à plus ou moins long terme d'une forme par l'autre, est celle qui a fondé la création d'éditeurs purement électroniques et qui a pu aussi habiter les anticipations d'un certain nombre de groupes multimédias, qui rapprochent la production des textes d'autres formes de la communication imagée, l'éloignant de la production imprimée. C'est aussi celle que l'on voit hanter les discours - que l'on pourrait dire à la fois prophétiques et performatifs -, d'un certain nombre d'entreprises, 
et en particulier les fabricants d'appareils - je pense à M icrosoft - , qui annoncent la mort prochaine, à la fois du livre, du journal, des revues dans leur forme imprimée, en scandant par une chronologie, comme ils l'ont fait au dernier congrès des éditeurs à Buenos Aires, cette substitution. On a donc là une seconde perspective qui est celle dans laquelle le monde du numérique apparaît, soit comme un soulagement possible à la crise de l'édition classique, soit comme, à terme, avec des diagnostics qui peuvent être à plus ou moins longue échéance, une substitution d'une forme de publication à une autre.

La perspective que j'aimerais analyser ici avec plus de détails est non pas la perspective sociologique, ni la perspective économique, mais la perspective historique, c'est-à-dire celle d'une histoire de longue durée de la culture écrite, qui essaye d'intégrer les mutations du monde numérique dans ce déploiement chronologique. Je crois qu'on peut et qu'on doit situer la révolution du numérique dans plusieurs ordres. Trois me retiendront: l'ordre des discours, l'ordre des raisons ou des raisonnements, l'ordre des propriétés.
L'ordre des discours

$C$ 'est évidemment un emprunt à un texte fameux de Michel Foucault. «L'ordre du discours » pour réfléchir sur la façon dont ont changé, jusqu'à cette mutation récente, les relations entre l'objet qui véhicule le texte, l'œuvre ou les œuvres qui sont portées par ce support, et la manière de les désigner ou de les assigner. C'est cette relation entrel'objet, l'œuvre et l'auteur qui me paraît tout à fait essentielle et qui, dans le monde qui est le nôtre, est le résultat d'un héritage de très longue durée à travers une série de sédimentations, et elle est profondément mise en question par le monde du numérique. Commençons par l'héritage. Je crois qu'il est très important de comprendre que le monde du texte écrit, et tout particulièrement des objets imprimés dans lequel nous avons vécu jusqu'à l'apparition du numérique - et dans lequel nous continuons à vivre très largement et d'une manière très majoritaire - , résulte de la sédimentation de quatre temps: le temps de l'objet, le temps de la technique, le temps de l'œuvre et le temps du nom.

- Le temps de l'objet
Il est clair que tous les objets de la
culture imprimée que nous 
connaissons ont une forme fondamentale qui renvoie à l'invention, au début de l'ère chrétienne, entre $I{ }^{\mathrm{e}}, \mathrm{III}^{\mathrm{e}}, \mathrm{IV} \mathrm{V}^{\mathrm{e}}$ siècles, d'une nouvelle forme de livre, celle que les historiens appellent « codex » et qui est formée de feuilles, quel que soit le matériau de ces feuilles, pliées une fois, deux fois, assemblées les unes avec les autres lorsqu'elles sont transformées en cahier et qui, du coup, donnent un livre qui est formé d'une série de cahiers, de feuillets, de pages, à l'intérieur d'une couverture et d'une reliure. $C$ 'est une rupture essentielle dans la mesure où le livre qui lui pré existait, le rouleau de l'Antiquité, répondait à des caractéristiques matérielles tout à fait différentes. Le rouleau de l'Antiquité est un livre sans pages, un livre sans cahiers, un livre sans index, un livre sans liberté corporelle du lecteur puisque, pour être lu, il doit être tenu à deux mains, ce qui fait qu'on ne peut pas écrire en lisant. Si on peut écrire, c'est parce qu'on a fermé le rouleau, et si I'on est en train de lire, les deux mains sont mobilisées pour tenir les deux supports autour desquels s'enroulent et se déroulent le rouleau. Cette révolution du codex est sans doute la révolution la plus fondamentale de la culture écrite après l'invention de l'écriture, quelle qu'elle soit, et avant le monde du numé rique, et tous les objets imprimés qui sont les nôtres ont une parenté fondamentale avec cette structure. Et donc, tous les gestes qui sont les nôtres, feuilleter - pour feuilleter un livre faut-il encore qu'il ait des feuillets -, repérer - grâce à des renvois de l'index au texte -, supposent qu'il y ait, là encore, foliotage, pagination, et possibilité d'indexation, ou le fait de pouvoir tenir le livre à distance, de le poser, ou de le tenir d'une main, lorsqu'il s'agit d'un format plus petit. Tous ces gestes essentiels, toute cette anthropologie de la lecture sont liée à l'invention du codex au pre mier siècle de l'ère chrétienne. Ce temps de l'objet est le temps le plus long, celui qui va du début de l'ère chrétienne jusqu'au $x x^{\mathrm{e}}$ siècle, peut-être qui sait jusqu'au XXII ${ }^{e}$ ou XXIII siècles...

- Le temps de la technique

À l'intérieur de cette durée, il y en a une qui est plus brève, mais Iongue néanmoins, que j'appelle le temps de la technique, c'est-à-dire le fait que les objets de lecture qui sont pour nous les plus nombreux relèvent de la technique de l'impri- 
merie, inventée au milieu du $\mathrm{XV}^{\mathrm{e}}$ siècle puis transformée avec I'industrialisation du $x x^{e}$ siècle. Ce qui veut dire ici que la copie manuscrite n'est plus la seule ressource possible pour transmettre un texte en de multiples exemplaires.

Deux précisions ici. La première renvoie aux travaux les plus récents de I'histoire du livre et souligne qu'on ne peut pas penser l'entrée dans l'âge de l'imprimé comme celui de la disparition de l'âge du manuscrit. Et qu'à l'âge de l'imprimé, du $x v^{e}$ jusqu'au $x x^{e}$ ou au $x x I^{e}$ siècles, les objets manuscrits restent nombreux, importants, et qu'entre le Xve et le xvIII siècles ils peuvent être les formes dominantes, majoritaires, de circulation d'un grand nombre de genres: les recueils poétiques, les nouvelles ou gazettes à la main, les manuscrits philosophiques. Cela constitue peut-être une leçon : une transformation technique peut ne pas être pensée en terme de substitution, et elle ne relègue pas nécessairement à l'oubli ou à la disparition la technique qui lui préexiste.

D euxième observation: vous voyez que je ne mets pas l'accent sur la comparaison qu'on peut lire souvent, dans une modalité savante ou dans une modalité journalistique, sur la comparaison entre le monde du numérique et de l'invention de Gutenberg. II me semble que le véritable accent est à mettre sur la comparaison possible entre les mutations profondes et radicales qu'impose la textualité numérique avec l'invention du codex, et non pas avec l'invention de l'imprimerie, parce que finalement l'invention de l'imprimerie n'a modifié en aucune manière, si j'ose dire, la forme fondamentale du support de l'écrit, et en particulier la forme du livre, telle qu'elle s'est mise en place au premier siècle de l'ère chrétienne. Et l'on voit que dans la révolution du numérique, ce n'est pas simplement la technique de composition ou de reproduction des textes qui change, mais fondamentalement les structures du support de la textualité, la forme donnée à ce texte sur l'écran et dans l'ordinateur, par rapport à celle qui est la sienne dans toutes les formes matérielles de la culture imprimée.

- Le temps de l'œuvre

Entre ce temps de l'objet et ce temps de la technique, il y a un autre temps qui est peut-être passé souvent inaperçu, et qui est le 
temps de l'œuvre. J'entends par là le moment où commence, pour des auteurs qui sont contemporains, cette manière de penser le livre, comme nous le pensons, c'est-à-dire à la fois comme un objet et comme une œuvre.

Demander à quelqu'un aujourd'hui s'il a lu le livre de Untel, c'est à la fois implicitement faire réfé rence à un objet matériel qui a cette forme du codex, mais c'est surtout faire référence à une œuvre identifiée à un livre. Et donc, du coup, il y a un lien étroit, absolu, entre le livre comme objet et le livre comme œuvre. Cette liaison n'est pas donnée d'elle-même, sauf pour les textes de la tradition chré tienne ou pour les textes de I'Antiquité, ou les corpus juridiques. Le livre en forme de codex au moyen âge est un livre de miscellanées, un livre de recueils, un livre en forme d'anthologie, réunissant une pluralité de textes de genres, de langues, de dates et d'auteurs différents à l'intérieur d'un même objet. Et il est fondamental de comprendre qu'au $X I V{ }^{e}$ siècle et dans la première moitié du Xve siècle, c'est-à-dire avant I'invention de Gutenberg, pour un certain nombre d'auteurs contemporains du temps, Pétrarque,
Boccace, Christine de Pisan, René d'Anjou, ou légèrement antérieur, $D$ ante, se met en place, à l'âge du manuscrit, ce lien étroit entre un objet matériel - livre - et une œuvre, c'est-à-dire que dans cet objet matériel on ne rencontre qu'une seule œuvre d'un seul auteur, ou plusieurs œuvres mais qui sont l'œuvre de ce même auteur. Et ainsi se met en place, dès avant l'invention de Gutenberg, cette association qui est pour nous presque automatique, incorporée, qui renvoie, fondamentalement, à une catégorie d'œuvres inscrites dans une matérial ité d'objets.

O n peut poursuivre en disant que la culture imprimée a renforcé bien sûr cette identité qui fait se lier étroitement le matériel et l'intellectuel ou l'esthétique; qu'en même temps elle a maintenu le genre des miscellanées, mélanges, anthologies, et que donc on aurait là encore des formes de persistance d'une structure de livres dominante à l'âge médiéval, peut-être minoritaire dans l'âge de l'imprimé, et qu'à l'inverse cette structure de livres que les Italiens appellent « libro unitario », le livre unitaire, dans lequel l'objet et l'œuvre sont identifiés, très minoritaire à l'âge du manuscrit pour les 
textes des contemporains, est deve nue une des formes dominantes de la culture écrite.

- Le temps du nom

À partir de quand une identitétextuelle est-elle référée fondamentalement à un nom propre, le nom d'auteur? Ce que j'ai dit précé demment en est une première pré figuration au XIVe et au XVe siècles. Avec la matérialité du livre unitaire, émerge cette figure d'auteur comme celle qui donne la clé de voûte de ce rapport entre une œuvre, ou une série d'œuvres, et un objet manuscrit; puis vient le temps des censures qui désigne les auteurs pour les interdire, les prohiber, ou les pourchasser - censure d'Église, censure d'État - et, au XVIII ${ }^{\mathrm{e}}$ siècle, le temps du copyright, le temps de l'invention de la propriété littéraire qui fonde les droits des libraires éditeurs sur ce droit premier de l'auteur sur son texte. C e sont ces moments clés qui vont identifier le texte à partir du nom propre, et ainsi prendre distance par rapport à d'autres régimes de circulation des textes, par exemple celui de l'anonymat.

Il faut donc bien comprendre que nos représentations, nos perceptions, nos catégories d'hommes de la fin du $x x^{e}$ siècle et du début du $\mathrm{XXI}^{\mathrm{e}}$ siècle, sont habitées, même inconsciemment, par ces durées longues: la durée du codex, la durée du livre identifié à l'œuvre, la durée de l'imprimerie et la durée de la «fonction auteur», pour citer un autre texte de Foucault.

$C$ 'est sur cet ensemble d'héritages sédimentés que la textual ité numé rique vient lancer ses défis ou, en tous les cas, vient produire des effets de désordre. D'abord parce que s'efface cette perception immédiate que nous avons entre des types d'objets, des classes de textes et des pratiques de lecture ou des usages de l'écrit. Le monde dans lequel nous avons vécu est un monde dans lequel il y a comme une immédiateté de cette association, un monde où des classes d'objets - le livre, le journal, le périodique, l'affiche, et si l'on déborde, l'imprimé, l'archive, la lettre, le journal personnel, etc. - , sont immédiatement perçus comme différents les uns des autres par leur matériau, leur format, leur forme, se lient à des types de textes, qui correspondent à l'un ou l'autre de ces objets imprimés ou manuscrits, et renvoient donc, du coup, à des rapports différents avec la culture 
écrite. L'ordre des discours est donc fondé sur la discontinuité des formes matérielles, immédiatement référées à des catégories de textes et à des pratiques de l'écrit et de la lecture, mais parfois au-delà même de la lecture.

Cette première réalité que nous pouvons énoncer conceptuellement, mais qui est incorporée en nous, qui est un système de perception immédiate, de représentation spontanée de la culture écrite, partagé par chacun, est profondé ment mise en question par le monde numérique qui est le monde d'un continuum textuel. Pourquoi? Parce que, d'une part, toutes les classes de textes si immé diatement distinguées par leur support matériel sont convoyées, véhiculées, données à lire par le même objet, l'ordinateur et son écran, et d'autre part, parce que ces textes de nature différente, véhiculés, donnés à voir et à lire sur cet objet unique, le sont dans des formes d'organisation, de présentation qui sont identiques ou quasi identiques, et qui sont en général voulues par les décisions du lecteur.

Cette opposition essentielle entre un monde de discontinuité maté rielle référée à des différences tex- tuelles ou à des pluralités d'usage d'un côté, et de l'autre côté, un continuum de textes qui est porté par un objet unique et qui donne à ces textes des formes semblables, a des conséquences, me semble-t-il, très profondes et qui, temporaire ment au moins, substituent à l'ancien ordre des discours ce que l'on pourrait appeler un désordre des discours si on le réfère aux catégories anciennes. D 'abord parce que s'effacent les distinctions entre les genres textuels. À partir du moment où la matérialité qui les porte et où les formes qui les organisent sont uniques ou semblables, les différences entre les genres textuels sont infiniment moins perceptibles que dans un monde où elles sont affirmées d'emblée par leur inscription dans des objets distincts.

Je crois que la pratique du collage si répandue avec l'ordinateur est comme le symptôme de ce transfert de textualités qui appartiennent à des genres, des registres, des formes extrêmement diverses, et qui circulent librement d'un lieu à l'autre par la volonté du lecteur. Effacement donc de l'immédiate distinction - immédiate parce que matérial isée - , entre les genres textuels, effacement aussi de l'immé 
diate perception de l'œuvre dans son unité ou sa totalité. Le continuum textuel qui est celui du texte numérique a évidemment pour conséquence le primat donné au fragment, c'est-à-dire à l'extrait qui vient découper une séquence dans cette continuité, et de là, sans doute, la perception de tous les textes en forme numérique sur le modèle des banques de données, ou pour le moins, la difficulté d'une perception de ce qui est la totalité de l'œuvre d'où sont extraits ces fragments. Alors que dans le monde de la culture imprimée, même si vous nelisez pas toutes les pages d'un livre, même si vous lisez un livre pour en extraire des citations, ce qui simpose à vous c'est l'unité, l'identité dans laquelle ces pages sont lues ou ces fragments sont extraits.

Un troisième effacement, qui n'est pas simplement l'effacement de la distinction entre les genres, I'effacement de la perception de la totalité de l'œuvre, réside dans l'efface ment de cette autorité différentielle qu'attribuait au texte le répertoire des formes imprimées.

Une illustration en est donnée par l'offre textuelle du web, du réseau, en particulier lorsqu'elle est maniée à travers les moteurs de recherche qui, à partir d'une entrée thématique, d'une catégorie, d'un mot clé, font saccumuler devant le lecteur des textes qui ont apparemment une cohérence et une unité puisque la forme qui les fait apparaître leur donne cette continuité, mais qui, évidemment, peuvent appartenir à des registres de textes dont l'autorité est profondément différente - et certains de ces textes étant sans autorité, voire dotés d'une autorité perverse. Le journaliste $D$ aniel Schneidermann analyse dans un ouvrage le résultat d'une recherche sur l'H olocauste ou la Shoah dans le monde du web, à partir d'un moteur de recherche, et il en conclut que ce qui apparaît, sinon majoritairement mais très massivement, ce sont les textes des sites révisionnistes, négationnistes, qui se présentent avec les mêmes critères, les mêmes formes, la même continuité textuelle que d'autres textes, qui eux proviennent de sites électroniques, de banques de données, etc. Et là on peut voir la différence: la même recherche menée dans le monde de l'imprimé, par exemple par un lecteur adolescent ou peu préparé, le conduira presque naturellement vers des classes d'objets, c'est-à-dire des classes de textes qui, à tort ou à 
raison - mais globalement à raison sont dotées d'une autorité scientifique, d'une valeur de contrôle, d'une garantie - encyclopédies, revues, manuels... D ans le secteur de l'imprimé, la propagande négationniste est prohibée dans un certain nombre de pays et de toute façon elle est d'un accès infiniment minoritaire, marginal, et secondaire alors que sur le web, une recherche menée par un moteur de recherche sur les thèmes Holocauste/Shoah, la fait apparaître comme importante, fondamentale, et pour considérer ce qu'elle est vraiment, manquent les critères d'identification de l'autorité différentielle des textes.

Tout cela pour dire qu'on est face à une situation devant laquelle les repérages immédiats, incorporés, qui étaient ceux des legs de la culture écrite et imprimée, sont mis en question: catégorie de l'unité du texte immédiatement visible à travers l'objet, catégories désignant la distinction des genres, rendus là aussi perceptibles par leur distribution entre des formes différentes, ou encore catégorie de l'autorité des textes qui renvoyait à des modes de publication, et donc du coup à des objets imprimés qui étaient de nature différente.
Le monde du numérique unifie, rapproche, assimile, ce qui ne veut pas dire que cela lui est un destin à tout jamais scellé, mais que l'un des défis du monde contemporain est évidemment de penser les catégories conceptuelles transformées en instruments ou dispositifs techniques qui sont capables de restituer, pour le lecteur - et en particulier le lecteur qui n'est pas formé, le lecteur ordinaire -, une possibilité de ces distinctions: perception de l'œuvre, distinction de genres, conscience des poids inégaux d'autorité des textes. Et c'est à ces conditions-là, je crois, que l'on peut reconstruire un ordre des discours à l'intérieur de la nouvelle forme qui leur est donnée.

\section{L'ordre des raisons}

Le second aspect que je voulais traiter concerne l'ordre des raisons ou l'ordre des raisonnements. Ce qui me paraît important ici, c'est une double mutation, permise par la textualité électronique: une mutation du côté des auteurs, une mutation du côté des lecteurs. D u côté de l'auteur, ce qui me semble fondamental, c'est la proposition d'une logique de démonstration qui ne soit plus enserrée dans les contraintes imposées par l'écrit. 
Les logiques de démonstration philosophiques juridiques ou historiques, dans les formes classiques de l'écrit, sont des logiques linéaires, qui sont celles de l'ordre d'une écriture déposéé sur une surface, une page, et à l'intérieur d'un objet tel que le livre, la revue ou le journal. On a donc une logique séquentielle et déductive qui est comme imposée par les formes classiques de l'écrit. À cela le monde du numérique peut opposer de nouvelles propositions qui sont celles de logiques éclatées par le jeu du lien, de logiques simultanées, faisant apparaître sur une même surface, en même temps, des éléments distincts et, par ce fait, une logique relationnelle d'un ordre nouveau par rapport à la logique déductive. II y a là tout un ensemble de réflexions qu'ont commencé à mener les logiciens ou les philosophes autour de figures comme celle d'une logique labyrinthique du côté de la logique permise par le texte numérique, opposée à une logique de la linéarité qui est celle de l'écrit en ses formes traditionnelles. C'est un premier élément du côté de la construction d'une argumentation. Vous voyez que cela peut renvoyer à des argumentations philo- sophiques, juridiques, ou des sciences sociales, à commencer par I'histoire.

Ce qui correspond à cela, du côté du lecteur, ce sont les nouvelles possibilités de réception, de validation, ou les critiques des démonstrations qui lui sont proposées. $D$ ans le monde du texte imprimé il y a comme une sorte de contrat implicite entre l'auteur et le lecteur; on pourrait appeler cela une structure fiduciaire, fondée sur la confiance de la preuve. Dans un livre de sciences sociales, par exemple un livre d'histoire, les conditions de la preuve renvoient à tout cet appareil critique qui est celui des notes, des citations, des références mais, évidemment, aucun des lecteurs ne refait le parcours qui est celui de l'auteur, et aucun ne va lire les textes des citations dans leur totalité, ou vérifier I'ensemble des références, ou se référer aux notes qui renvoient à des documents d'archives. II y a donc là une sorte de pacte de confiance entre l'auteur et le lecteur.

Le monde du numérique, à la condition évidemment que les documents eux-mêmes aient été numérisés, ouvre des possibilités tout à fait inédites. D 'une part, il 
peut mettre le lecteur face à la totalité ou partie de ce qui est l'objet même de la démonstration - documents d'archives, textes anciens imprimés -, et donc lui donner des critères ou des conditions de validation du raisonne ment et de la démonstration qui sont proposés - qui sont évidemment au plus près de ce sur quoi elles portent, puisque le lecteur lui-même peut refaire tout ou partie du chemin, d'une manière qui peut lui prendre du temps mais qui est techniquement possible et d'autre part, ce qui est présent, là, face au lecteur, ne consiste pas simplement en des textes d'archives, ou en des textes imprimés, mais peut relever d'autres ordres qui sont ceux de l'image, y compris l'image mobile ou le son - musique, paroles.

Et l'on voit alors que cette mutation du numérique du côté de l'organisation des démonstrations et du côté de la validation de ces argumentations doit être considérée comme une mutation épisté mologique. C'était l'autre aspect de cet article de Robert $D$ arnton que de montrer que, non seulement, les livres de savoir pouvaient trouver des manières d'être publiés, alors que les éditions imprimées s'en détournaient, par la forme de l'édition numérique, mais aussi que les auteurs et les lecteurs devai ent exploiter les possibilités nouvelles qui leur étaient offertes: pour les premiers, construire leur argumentation de manière inédite et, pour les seconds, exercer leur jugement, leur appréciation critique avec des instruments plus nombreux, plus divers, et finalement au plus près de ceux que I'historien a manié. II en a donné un exemple dans un article récent, qui est publié sous formes imprimée et électronique, et qui est consacré au rôle de la chanson dans le Paris du XVIII ${ }^{\mathrm{e}}$ siècle comme forme de cristallisation de l'opinion publique. Et l'on voit bien la différence puisque le «même » article, lorsqu'il est rendu accessible sur le site de l'American $\mathrm{H}$ istorical Association, permet au lecteur d'avoir accès à la totalité du corpus des chansons qui sont analysées, de suivre une cartographie dynamique des lieux successifs où l'on a utilisé la chanson comme forme de mobilisation de l'opinion publique dans le Paris du XVIII e siècle, et égal ement d'entendre les chansons puisqu'elles ont été enregistrées par H élène D elavault, sur la 
base à la fois des manuscrits de la bibliothèque de l'Arsenal - archives de la Bastille - et des airs connus du XvIII siècle auxquels renvoyaient ces chansons.

Voilà pour l'ordre des raisons, et vous voyez que je me garde de tout diagnostic parce qu'il me semble que la discussion sur le numérique a été trop longtemps comme enserrée entre des positions convenues: d'un côté, par ses adversaires, la déploration du monde de l'écrit perdu et de l'autre côté, par ses prophètes, des enthousiasmes utopiques de l'entrée immédiate et universelle dans une nouvelle civilisation. Je pense que ces discours-là ont peut-être fait le profit de leurs auteurs, mais n'ont pas éclairé beaucoup la réflexion. Ce qui est important, me semble-t-il, c'est d'essayer de porter des diagnostics qui soient fondés sur des observations analytiques. Parfois, si on les traduit en termes plus psychologiques, ils peuvent amener à penser que ce désordre introduit dans notre ordre des discours conduit à certaines formes de désarroi. $M$ ais, à l'inverse, cette possibilité nouvelle de la production de la réception des démonstrations peut conduire à une excitation ou à une incita- tion intellectuelle plus ou moins forte chez les uns et chez autres.

\section{L'ordre des propriétés}

Je vais évoquer maintenant l'ordre des propriétés. J'emploie le mot «propriétés » dans deux sens: I'un que vous pouvez attendre, c'est-àdire le sens juridique et économique de la propriété littéraire, et puis l'autre plus formel, esthétique ou intellectuel, qui est celui de la propriété des textes eux-mêmes, non pas la propriété sur les textes, mais les propriétés des textes. Parce que les deux me paraissent étroitement liés.

II est clair que jusqu'à présent notre perception et nos emplois ont utilisé ou apprécié la textualité numérique à partir de catégories comme celles de mobilité, de malléabilité, d'ouverture. Le monde du texte numérique est un monde où les textes sont déployés, repris, réécrits, où une écriture s'écrit dans une écriture déjà là, un monde où le lecteur intervient non pas sur les marges du texte, mais dans les textes eux-mêmes, un monde où comme l'avait rêvé parfois Foucault, s'effacerait l'assignation au nom propre, où s'effacerait la «fonction auteur » dans une sorte de textualité formée de 
nappes de discours toujours repris et liés à l'échange permanent entre producteurs et lecteurs - mais des lecteurs à leur tour auteurs.

Ce mondelà de textes mobiles, malléables, ouverts, lance un défi sérieux aux catégories de description et d'attribution des œuvres telles que nous en avons héritées, l'œuvre comme une identité qui a sa clôture, l'œuvre qu'elle soit intellectuelle, esthétique, quel qu'en soit l'ordre scientifique, comme ayant une intégrité qui la rend identifiable comme telle, et l'œuvre rapportée - j'en ai marqué rapidement les étapes - à un nom propre. La perception et les usages de la textualité numérique se sont construites quasiment en opposition, terme à terme, par rapport à ces critères qui définissent l'œuvre dans le monde de l'imprimé. Là, nous sommes du côté de la propriété des textes, mais l'important est le fait que ce sont ces critères qui rendent identifiables l'œuvre - parce qu'elle est close, parce qu'elle est stable et parce qu'elle est attribuable -, qui ont fondé, à partir du XvIII siècle, les catégories juridiques qui dominent le monde de l'écrit et, en particulier, la catégorie de propriété litté raire. La propriété littéraire est entendue au sens de la propriété imprescriptible de l'auteur sur son œuvre, parce que cette œuvre identifiable comme telle est irréductiblement liée à ce geste créateur qui est celui de l'écriture et, du coup, s'effectue le transfert de cette propriété à celui qui achète le texte et qui en est l'éditeur. Tout le régime juridique qui assure à la fois la protection du droit patrimonial et du droit moral de l'auteur, et la rémunération ou le profit lié au travail d'édition, renvoie à cet ensemble de catégories qui, à partir du XVIII ${ }^{e}$ siècle, a défini ce qu'est l'identité d'une œuvre. $M$ ais toute identité reconnaissable suppose des critères de délimitation, d'assignation, et de stabilité. Or le monde du numérique que nous pratiquons, que nous connaissons, majoritairement, est ce monde dans lequel les critères sont opposés - malléabilité, mobilité, ouverture. Je crois que la tension ou la contradiction naît de ce que nous voyons apparaître: un monde numérique tout différent de celui auquel les premiers temps de I'Internet, du réseau, des site web, nous ont habitués, c'est-à-dire un monde numérique dans lequel les textes retrouvent fixité, stabilité, fermeture, et qui est le monde 
recherché, peut-être nécessaire, pour l'édition numérique. II faut distinguer ici entre ce qui serait une publication - rendre publics des textes électroniquement, ce que peut-être certains font ici, et que nous faisons peut-être tous d'une certaine manière, et transmettre des textes à des lecteurs, plus ou moins nombreux, d'une façon libre, gratuite, spontanée et une édition électronique, qui suppose les opérations classiques de l'édition et qui rend possible le paiement du travail éditorial et de la création intellectuelle ou esthé tique. C'est comme cela, je crois, qu'on a vu se mettre en place toute cette réflexion autour de machines ou d'objets qui, dans le monde du numérique, à la différence des ordinateurs classiques, de forme $P C$, ferment les textes, les rendent stables, mais en même temps les rendent immobiles, c'est-à-dire ne permettent pas qu'on les transmette, qu'on les copie, voire qu'on les imprime.

De là, la recherche de machines par les fabricants, ou par un certain nombre d'éditeurs en ligne autour de l'« e-book » - bien mal nommé puisqu'il ne sagit pas d'un livre, mais soit d'une bibliothèque qui comporte divers textes, soit d'une sorte d'agenda dans lequel un lecteur peut inscrire ce qui lui est personnel -, qui a pour caractéristique de transmettre au lecteur des textes qui s'affichent soit parce qu'ils ont été téléchargés, soit parce qu'ils proviennent d'un microprocesseur, mais qui de toute façon ne permet pas que ces textes soient transmis, copiés ou imprimés. Et de là un effet paradoxal, ambigu - je me garde de juger puisque, d'un côté, cela peut être la condition de protection de l'identité des œuvres, donc du droit des auteurs et des profits des éditeurs et que, d'un autre côté, il est clair que cette textualité électronique de l'édition électronique liée au « e-book » est opposée, terme à terme, à ce qui pour nous a fait le plaisir ou l'intérêt de la technique électronique, c'est-àdire la malléabilité, la mobilité, l'ouverture.

A partir de là, on voit qu'il y une double interrogation, peut-être une double hypothèse sur le futur du numérique. Première interrogation et hypothèse: ne va-t-on pas vers une sorte de partage dans le monde du numérique entre deux formes de textualité? L'une qui assure la protection des textes, c'est-à-dire la protection des droits 
et des profits, et qui se liera à toute une famille d'objets qu'on va appeler "e-book», même sils peuvent porter des noms différents, qui renvoie à un travail d'édition, qui renvoie à une position d'auteur, et qui renvoie au marché. Et, de I'autre côté, une textualité numé rique qui nous est peut-être plus familière, qui est celle des textes mis en libre circulation, de façon gratuite, avec les critères d'ouverture et de mobilité qui les caractérisent, qui seraient véhiculés sur les ordinateurs classiques tels que nous les connaissons, et qui renverraient à la communauté, à l'échange, au partage. Je ne sais pas si ce diagnostic est juste mais en tous cas la question doit être posée sur les rapports entre publication numérique et édition numérique, puisque des familles d'objets correspondent à l'une ou l'autre de ces deux modalités, et que les propriétés (au deux sens du terme: propriété des textes, propriété sur les textes) se distribuent différemment selon l'une et l'autre de ces formes.

D euxième interrogation et hypothèse: est-ce que nous devons penser cette réflexion sur le livre et le numérique, ou le livre numérique, ou la textualité numérique, comme je l'ai fait jusqu'à maintenant, en rapport exclusivement, nécessairement, avec l'ordinateur quel qu'il soit, c'est-à-dire l'ordinateur de la famille PC, le portable ou le «e-book »- ou est-ce que, dans un avenir plus ou moins proche, pour la première fois, la communication des textes électroniques ne pourrait-elle pas se détacher des objets que sont les ordinateurs? Je fais allusion à ces recherches expérimentales qui ont conduit à l'invention d'une encre et, entre guillemets, d'un papier électronique, c'est-à-dire d'un support sur lequel peuvent apparaitre des textes électroniques, soit venant d'un microprocesseur, soit télé chargés, et qui peuvent être déposés - ce papier doté de cette encre -, sur n'importe quelle surface, que ce soit des surfaces de matériaux différents, de tailles différentes, de localisations différentes, y compris, pourquoi pas, des livres en forme de codex tels que nous les connaissons.

Certains historiens de la Rome antique, alors que l'on a souvent parlé de la trajectoire qui mène du rouleau au codex, ont voulu remettre en question ce schéma trop simple en disant que, finalement, avant les rouleaux existaient 
dans l'ancienne Rome des livres faits de tablettes associées, qui étaient des sortes de " protocodex ». Et, comme un défi aux idées reçues, ils ont proposé l'idée qu'il y avait une trajectoire qui allait du codex au rouleau, avant, dans un second temps, d'aller du rouleau au codex. Si I'on suit les suggestions de ces recherches pour notre présent, on aurait en quelque sorte, non pas simple ment la trajectoire quej'ai dessinée qui nous mènerait du codex à l'écran, un écran qui se substitue totalement, ou un écran qui prend place à l'intérieur d'un monde dans lequel le codex imprimé reste présent, majoritaire ou minoritaire - cette question est indécidable - , mais on aurait, éventuellement, une trajectoire qui irait de l'écran au codex, si I'on entend par là que

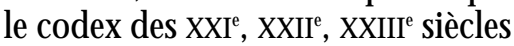
sera doté de ce papier et de cette encre électroniques et gardera quelque chose de la forme de livre inventé au début de l'ère chrétienne - qui, dans ce cas, aurait une robustesse ou une résistance plus grandes que celle quel'on imagine. Après tout, le codex a été le support d'abord d'une écriture manuscrite et ensuite d'une écriture imprimée, en même temps que pouvaient demeurer des usages du manuscrit. Pourquoi ne pas penser que le numérique et le codex peuvent un jour se rencontrer?

Merci pour votre attention. Je serais heureux maintenant quel'on puisse entrer dans une discussion.

\section{Public}

Vous avez évoqué le livre au début de l'ère chrétienne. D e quelle façon pensez-vous que ce livre se présentait à cette époquelà?

\section{Roger Chartier}

Je suis un historien des $x v I^{e}$,

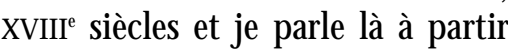
de la lecture des travaux des spé cialistes de l'Antiquité. Le début de l'ère chrétienne est marqué justement par un mode de coexistence - ou peut-être de compétition entre la forme ancienne qui est celle du rouleau - et Iorsqu'on a affaire à des œuvres qui ont une certaine ampleur, des rouleaux qui correspondent à une œuvre - et, d'autre part, l'apparition des premiers codex, donc de ces livres tel qu'un livre actuel peut plus ou moins nous en donner une idée, surtout s'il s'agit d'un grand format. Nous avons donc là une coexistence qui, progressivement, va voir la domination de la forme 
codex sur la forme rouleau pour des raisons qui sont largement religieuses, et le fait de la rapporter au christianisme n'est pas fortuit. Bien sûr il y a toutes ces raisons que j'ai évoquées, c'est-à-dire que la pratique de lecture du codex est, pour le corps, une pratique plus libre, plus à distance du livre - il peut être posé, être porté, on peut avoir les mains libres - , et d'autre part c'est une pratique qui, intellectuellement, permet les repérages, puisque si vous avez un foliotage ou une pagination vous pouvez établir un index des citations, des références, des noms propres, etc. Donc le codex présente intellectuellement et corporellement des avantages par rapport ce qu'était le rouleau, ce qui assure son succès.

En même temps, par rapport aux usages chrétiens, il est sûr que pour une religion qui est fondée sur la comparaison entre les Écritures - Ancien et Nouveau Testament, Évangiles -, et sur I'utilisation liturgique des fragments du texte sacré, la forme du codex est particulièrement adaptée à cette double relation du christianisme avec l'écrit. Les comparaisons ou confrontations ont porté l'élaboration des concordances qui ont très vite été liées au codex manuscrit et le repérage du fragment, de l'extrait, de ce qui deviendra plus tard des versets bibliques, a été aussi rendu infiniment plus aisé avec le codex. II y a donc à l'intérieur de cette proposition, une forme de livre qui est intellectuellement plus maniable, et qui est corporellement à distance du corps, qui lui laisse plus de liberté; et il y a certainement - et c'est pourquoi on peut établir que c'est dans les communautés chrétiennes que l'usage du codex s'est mis en place le plus rapidement - , comme une adéquation entre le rapport au texte sacré dans le christianisme et la forme codex.

\section{Public}

Accepteriez-vous comme définition du codex la réunion de ce qui, auparavant, était des tablettes séparées?

\section{Roger Chartier}

C'est une grande question. Entre les tablettes et le codex il y a ce monde que nous avons du mal à imaginer parce que les témoignages archéologiques sont rares, qui est ce monde des rouleaux, des volumina, et dont on a quelques extraits; mais rarement sous forme 
complète, sauf très tardivement, et pour le seul site où des rouleaux ont pu être conservés de manière assez intègre, qui est le monde égyptien, un monde où les conditions climatiques et le sol l'ont permis. N ous avons toujours lu c'est un nous collectif qui commencerait peut-être avec les hommes d'O ccident du $x^{e}$ siècle-, les textes de l'Antiquité dans une forme codex, depuis les manuscrits médiévaux qui ont transmis la tradition du texte jusqu'à nos éditions Budé ou nos livres de poche. Pour nous la littérature, la philosophie antiques sont dans la forme du codex: index, pages, tables. II faut imaginer que les auteurs ou les lecteurs de l'Antiquité ont eu un rapport totalement différent avec ces textes philosophiques ou littéraires, à l'intérieur d'un monde de l'écrit qui était celui des rouleaux, ce qui avait par exemple pour premier effet, si l'œuvre était trop longue, de la disséminer entre une plusieurs rouleaux. II n'y avait pas d'unité entre l'objet livre et I'unité œuvre. II faut encore imaginer un rapport à ces textes qui n'est pas du tout fondé sur les techniques de repérage, de localisation tels que les index les rendent possible. II y a donc tout un effort d'imagination à faire pour penser ces œuvres dans la matérialité qui a été la leur, et cette matérialité se situe, par rapport à votre question, entre le monde des tablettes et le monde du codex. Certains des spé cialistes de la philosophie antique le font, bien évidemment: des philologues tel que Jean Bollack, un historien de la philosophie espagnole comme Emilio Lleidó, par exemple. La matérialité du rouleau est essentiellement le papyrus, mais pas forcément: il y a des rouleaux sur parchemin, et il y a des codex sur papyrus, et les manuels vont trop vite quand ils opposent toujours le rouleau de papyrus au codex sur parchemin, et ensuite sur papier; mais dans les témoignages archéologiques ce n'est pas le matériau qui fait la différence, c'est la structure de l'objet.

\section{Public}

En tout cas, pour la maniabilité du document, la tablette numérotée ne pose absolument aucun problème, et le plus ancien dictionnaire actuellement existant qu'on ait retrouvé à l'heure actuelle, les tablettes d'Eridu, qui présente l'alphabet araméen et des textes en araméen, se présente comme des tablettes séparées les unes des 
autres, qu'il est parfaitement possible de numéroter, et qui procurent la commodité d'une pagination numérotée. Simplement les «pages», si l'on peut dire puisqu'il sagit là de tablettes d'argile, sont séparées les unes des autres.

\section{Roger Chartier}

Vous n'êtes pas loin de l'argument de Guglielmo Cavallo que j'évoquais, qui pense que l'on va du codex au rouleau parce que des tablettes différentes associées I'une à l'autre, comme on le voit sur certaines fresques de Pompéi ou $d^{\prime} \mathrm{H}$ erculanum ont quelque chose à voir avec le codex. Le rouleau qui serait à Rome une importation et une imitation du monde grec. $C$ 'est vrai que le monde des tablettes est dans une forme « proto-codex » à la grande différence du monde du rouleau, rouleau de papyrus majoritairement, rouleau sur parchemin minoritairement.

\section{Public}

J'ai été très intéressée quand vous avez parlé d'une certaine contradiction entre le livre, le e-book, assez fermé, et la communauté de la recherche. Est-ce que vous avez des idées sur la façon dont la recherche pourrait être affectée par ces mutations fondamentales et comment voyez-vous le fonctionnement de la recherche dans l'esprit d'une communauté de l'Internet? D'autre part, pourriezvous nous parler un peu plus des index, parce que ça me paraît être un élément assez fort de la recherche aujourd'hui dans les documents numériques et une modification fondamentale dans la façon de travailler?

\section{Roger Chartier}

La communauté de la recherche par rapport à l'édition électronique peut être entendue de plusieurs manières. II y a une premiè re manière qui est une manière documentaire dont le lecteur du livre d'histoire qui serait face à tout ou partie des textes qui sont l'objet même de l'analyse, peutêtre une figure amplifiée. II est clair que la première manière dont les chercheurs, quelle que soit leur discipline, au moins dans les sciences humaines ou sociales, ont utilisé le numérique, c'est en terme de l'immédiateté des ressources, et de la possibilité de recevoir bibliographies, catal ogues et documents. II faut rappeler que, malgré cette facilité d'accès qui supprime le 
temps et l'espace, dès que l'on entre dans un rapport avec des textes anciens - que ce soit des documents d'archives numérisés ou des éditions anciennes numérisées -, on ne doit pas supposer que I'on a affaire à un substitut strictement identique à ce à quoi il se substitue. La matérialité de l'objet écrit dont j'ai parlé, définissant son identité, et de ce fait, historiquement, définissant les usages ou les interprétations qui ont pu en être faites, reste essentielle. Je crois qu'il y aurait un très grand danger, qui correspondrait à une très grande paresse, à penser que parce que la numérisation permet de recevoir des images des pages d'une édition ancienne ou des documents d'archives, on pourrait éviter la rencontre avec la matérialité première dans laquelle ces textes ont été inscrits. Et c'est là une précaution absolument fondamentale de penser que le substitut est un substitut, à la fois dans sa force de pré sence et en même temps dans les limites de son adéquation à l'objet. $C$ 'est un peu « ceci n'est pas une pipe »: une édition du $x v I^{e}$ siècle numérisée n'est pas l'édition du $\mathrm{XV} \mathrm{I}^{\mathrm{e}}$ siècle. II y a une nécessité à le rappeler, et cela fonde d'ailleurs le rôle des bibliothèques d'une manière particulièrement forte; en particulier, de toutes les bibliothèques - elles sont nombreuses en France, universitaires, municipales, nationale -, qui ont des fonds patrimoniaux et qui, du coup, ont la fonction de rendre accessible dans la matérialité pre mière ce que, par ailleurs, la transmission numérique peut rendre transmissible à distance. L'accès aux formes numériques du patrimoine écrit, qu'il soit manuscrit ou imprimé, doit être démultiplié, mais avec cette précaution fondamentale qui consiste à reconnaître l'apport fondamental de ce qu'on pourrait appeler la bibliographie, au sens de comprendre les textes dans leur identité « corporelle». On peut penser ensuite à ce que j'évoquais, c'est-à-dire à la recherche utilisant des supports aux modes d'argumentation qui sont propres à la textualité numérique, et impossibles dans la textualité imprimée ou manuscrite, et à ce que vousévoquez qui est une dimension qui ne serait plus documentaire, qui ne serait plus démonstrative, mais qui serait communautaire, c'est-àdire des communautés de recherche assurant la communication, se faisant en quelque sorte éditeurs ou éditrices d'elles-mêmes, se soustrayant aux 
Iois de ce qui serait une édition électronique- jecrois que dans certaines disciplines, cette formelà de communauté savante existe déjà. Les communautés scientifiques dans le domaine des sciences dures ont sûrement été les premières à expérimenter ce type de relations et, finalement, à se fonder sur ce mode de l'échange des textes, puisque même sil sagit de démonstrations mathé matiques il sagit bien de textes, et peut-être qu'une voie possible de développement est que les communautés de sciences humaines et sociales le fassent à leur tour.

Le problème est assez complexe. AuX XVII et XVIII ${ }^{e}$ siècles, le manuscrit était la manière de fonder et de donner existence à la République des lettres par l'échange de lettres, l'envoi de mémoires, la copie de documents. Entre 1650 et 1750, on a ainsi un monde de savants et d'érudits qui est défini par une éthique de la gratuité, du désinté ressement et qui a le manuscrit pour véhicule. II est évident, qu'en même temps, ces savants et ces érudits publiaient, et publiaient sous une forme qui était celle de l'imprimé, et entraient de ce fait dans une autre logique, la logique des libraires éditeurs, la logique du marché, la logique du commerce du livre. Peut-être que la situation à laquelle nous pensons, vous et moi, serait un peu de ce même ordre: des communautés scientifiques qui se fonderaient sur ces modes de l'échange permis dans leur immédiateté et leur gratuité par le monde du numérique échange d'informations par le courrier, proposition de textes à la critique, circulation de documents... M ais en même temps, à partir du moment où le savoir scientifique doit être un savoir validé et public, il me semble qu'il est difficile de faire l'économie de ce qu'on pourrait appeler une forme d'édition électronique, c'est-à-dire une forme de contrôle, une forme de validation. Ce qui est en jeu, peut-être, c'est que la fonction d'édition n'est pas nécessairement assumée par des acteurs sociaux qui sont ceux que nous connaissons comme éditeurs, et l'on voit évidemment des revues portées par des communautés savantes qui sont éditées numériquement, donc qui assurent directement choix, tri, sélection, contrôle, validation.

Est-ce que pour les sciences humaines et sociales cette sorte de réappropriation totale est souhaitable ou possible? Je ne sais pas, dans la mesure où du côté des 
sciences humaines et sociales le fait de passer par l'édition, le commerce du livre, le marché a toujours été aussi lié à la production du savoir, ce qui n'est sans doute pas le cas du monde des mathématiciens ou des physiciens pour lesquels il n'y a pas de public en dehors d'eux-mêmes, dans leurs formes les plus extrêmes de scientificité. Pour un historien ou pour un sociologue, il y a un public audelà de la communauté des historiens ou des sociologues.

Est-ce qu'il serait souhaitable ou possible, y compris dans le monde du numérique, avec ses possibilités, que la diffusion des résultats de recherche auprès ce public soit uniquement portée par la communauté? O u ne va-t-elle pas, à un moment donné, rencontrer l'édition électronique et, par là même, des spécial istes de cette opération? Je ne sais pas. $M$ ais en tous les cas, sur le registre documentaire, sur le registre démonstratif et le registre communautaire, il me semble qu'il y a là des possibilités déjà exploitées, mais que l'on verra, sans nul doute, se multiplier.

En ce qui concerne la question des index, évidemment on est passé, en fonction des trois états dont nous venons de parler, rouleau, codex - à l'intérieur du codex, codex imprimé - et maintenant textualité numérique, à une démultiplication deleurs potentialités. Comme je le disais, on ne peut pas indexer un rouleau, parce qu'il n'y a pas de systèmes de repé rage et de distinction de la page ou du feuillet qui le permettent. Le codex manuscrit permet des indexations, mais qui ne sont liées qu'à l'objet particulier, puisqu'il n'y a pas d'identité absolue d'un codex à l'autre. Le codex imprimé permet une standardisation, c'està-dire des index qui valent pour tous les exemplaires d'un même édition. Pour palier cette difficulté, à l'époque médiévale, on essayait d'indexer, mais non pas en référant à des pages ou à des folios du livre mais à des parties chapitre ou des zones du texte, ce qui était une manière d'avoir des index alors même que la matérialité du livre était différente. Évidemment, avec la textualité électronique, l'index, c'est-à-dire tout ce qui permet les repérages, les consultations, les traversées, multiplie sa puissance. Pour prendre un exemple, il existe maintenant deux éditions numé riques de l'Encycl opédie de D iderot et d'Alembert: une édition réal isée par l'université de Chicago et une 
édition commerciale. Et il est sûr que la lecture actuelle de cette encyclopédie est peut-être celle que souhaitaient Diderot et d'Alembert, mais en tout cas ce n'est pas celle que pouvaient en faire les lecteurs du XvIII ${ }^{e}$ siècle: la durée de la publication, la relative difficulté de manipulation du livre, la longueur et la lourdeur des opérations, tout le système de renvois tel qu'il était construit par les promoteurs de l'Encyclopédie, la rendait en effet très difficilement maniable. Aujourd'hui on peut évidemment, immédiatement, en un instant, avoir accès à tout le système explicite des renvois que les éditeurs ont déposé à la fin des articles - et vous vous souvenez que c'est souvent par le jeu des renvois qu'apparaît le contenu le plus critique du livre; c'est lui qui introduit l'ironie alors que I'article est particulièrement classique ou orthodoxe. Ce jeu des renvois, si lourdement maniable au XVIII e siècle, devient donc immédiatement accessible aux lecteurs d'aujourd'hui. L'exemple de l'Encyclopédie me paraît un exemple tout à fait topique de cette histoire, que j'ai essayé de retracer, à très gros traits, de la potentialité toujours accrue de
I'index, c'est-à-dire le fait d'entrer dans des textes, non pas à partir de leur déploiement séquentiel, mais à partir de la traversée que permet l'indexation.

\section{Public}

II faut préciser que la production de documents scientifiques à travers les réseaux, et notamment la production de documents publics, est de plus en plus généralisée et que ces documents sont accessibles à travers le réseau de façon totalement gratuite. Le gouvernement promeut de plus en plus la production de documents publics gratuits sur le web à travers les différents sites gouvernementaux, y compris ceux del'Assemblée national e et du Sénat, ce qui permet aujourd'hui d'avoir quantités de séances retranscrites, directement accessibles grâce au web alors qu'il n'y a pas si longtemps il fallait aller à la Documentation française pour avoir ces mêmes documents et en payer une copie. Ce qui est toujours le cas d'ailleurs, puisque si les documents sont gratuits sur le web, ils sont payants quand ils sont imprimés. Ce qui pose d'ailleurs une question de fond: si les documents sont payants sur le papier c'est tout simplement que le papier 
coûte et une des grandes différences entre le document électronique et le document papier, est liée à des questions essentiellement de coût et ce n'est pas négligeable parce que cela va induire des modèles économiques très diffé rents. C ela m'amène à la question que je voulais aborder. Tout à I'heure, vous parliez de ce monde de la publication qui est en train de se scinder en deux: d'un côté des documents plus ou moins libres de droit sous un système de référence dit de logiciel libre, ce qui fait qu'effectivement les auteurs donnent libre accès à des documents avec accès à la modification et à la republication éventuellement de ces documents sans demande, autorisation ou rémunération -, et des documents qui peuvent être tout simplement librement diffusables, ce qui est autre chose: c'està-dire que les auteurs publient, mais en gardant leur droit moral et en interdisant que ce document puisse être diffusé avec modification; et puis de l'autre côté ce monde de la diffusion du document qui est beaucoup plus contrôlé, tout simplement parce que les structures qui les diffusent en attendent du profit. C'est aussi un moyen, pour le monde de l'édi- tion, de sauver les meubles, et d'essayer de trouver une porte de sortie ou une porte d'entrée, ça dépend dans quel sens on le prend, vers le monde du numérique, tout en sauvegardant ses intérêts ou les marchés qu'il a déjà pu acquérir sur le monde du papier. Là où je suis un peu moins d'accord avec vous c'est lorsque vous opposez la liberté d'accès à des documents sur des ordinateurs classiques - PC ou $M$ acintosh -, à un univers du document plus fermé qui serait récupéré sur des machines portables. À mon avis il n'y a pas de fatalité à ce que des machines portables puissent également récupérer des documents ouverts, ou du moins à ce que des documents puissent se transmettre de machine à machine, ou à ce que des documents qui circulent librement sur le web puissent être récupérés par des machines portables. Aujourd'hui il y a peu de machines encore sur le marché, mais même les machines qui ont un petit écran type palm ou les machines type pocket PC, marchant avec le systè me du palm os qui commence à se diffuser, et de l'autre côté les pockets PC fonctionnant avec les systèmes diffusés par M icrosoft, n'empêchent pas pour autant une diffu- 
sion de plus en plus large de documents de machine à machine. $\mathrm{Ce}$ qui, en terme d'usage, est quelque chose d'inconnu dans le monde classique du papier. Q uand on a un livre en papier dans la main et qu'on veut le communiquer à quelqu'un, il faut aujourd'hui se dessaisir del'objet et le lui donner. À partir du moment où des machines communiquent entre elles très facilement - et cela existe - , quand on lit un document et que quelqu'un a la même machine ou une machine compatible, on peut le lui transmettre en quelques secondes, et il pourra le lire dans les mêmes conditions, qu'il soit chez lui, ici ou dans un hall de gare. II n'y a donc pas de fatalité liée à la diffusion des documents et au type de machines. Le débat me semblet-il qui devrait plutôt mobiliser notre réflexion, a trait aux nouveaux modèles économiques qui vont être mis en œuvre pour que les auteurs et les éditeurs, éditeurs au sens large des structures éditoriales, qui ne sont effectivement pas nécessairement les éditeurs qu'on connait aujourd'hui, puissent vivre de la diffusion de documents électroniques. Aujourd'hui personne n'a réellement la réponse à cette question, et puis - corollaire à cela -, quelle est la valeur économique d'un document électronique? Aujourd'hui on connaît la valeur économique d'un livre papier: elle est en gros lié au coût de fabrication de cet objet en papier, et il y a un lien direct entre les coûts de fabrication et les coûts des livres; mais quelle va être la valeur d'un bien culturel électronique qui, lui, n'a pratiquement pas de coût de production, et qui a des coûts de distribution quasiment nuls? C'est une grande question à laquelle je ne sais pas si on peut apporter des réponses.

\section{Roger Chartier}

Vous avez absolument raison de dire qu'il n'y a pas de fatalité des machines - appelons-les «e-book » pour faire vite -, qui ferait qu'elles ne seraient que le support de textes qui relèveraient du marché des textes, et vous avez, ayant plus de compétences que moi, tout à fait raison de dire que ces machines-là aussi peuvent être aussi au service de cette communication libre, à tous les sens du mot, des textes. Je me plaçais du point de vue des éditeurs, comme M icrosoft, pour qui le monde de l'accès payant aux textes est un monde qui est toujours mis en danger par le piratage 
lorsqu'il se déploie à travers les PC ou les $M$ acintosh. Et le fameux exemple de Stephen King, si souvent cité - les deux chapitres du premier texte -, est là pour montrer qu'il y a à la fois un appétit de lecture sur écran - puisqu'il y a eu d'aussi nombreuses demandes mais en même temps que ceux qui ont piraté le texte - puisqu'à l'époque il était édité par un éditeur et qu'il devait être payant, même modiquement -, l'ont fait parce que le texte pouvait être accessible sur PC et donc, ce qui devenait fondamental était de trouver des modes de transmission de ces textes où le piratage soit sinon impossible, du moins très limité. Ce que j'ai entendu - mais je n'ai pas de compétences techniques - , c'est que dans la perspective des éditeurs, tout ce qui est transmission des textes sur les ordinateurs de type classique, est toujours sujet à piratage, alors que l'encryptage du «e-book», et l'impossibilité - même lorsque le texte est téléchargé à partir de l'Internet et donc passe par un PC -, de le garder sur la mémoire du disque dur, fait qu'un lien s'établit, quasi automatique, entre les machines qui évitent théoriquement le piratage et le commerce des textes élec- troniques. Vous avez absolument raison: ces «e-book » ne sont pas nécessairement support de textes fermés, mais pour les « éditeurs » qui proposent ce marché du texte électronique, ils sont la condition d'une protection de ce qu'ils disent, à juste titre, être les droits des auteurs, mais aussi, de leur propre rémunération.

Vous avez raison aussi de parler du coût, et il est effectif que même si on n'est plus dans la situation du XVIII ${ }^{\text {e }}$ siècle, le papier reste pour une édition un chapitre lourd. Vous savez peut-être qu'au

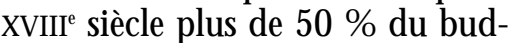
get d'un livre était consacré au simple achat du papier. Ce qui est peut-être une logique à l'œuvre, c'est ce transfert des coûts, puisque la plupart des textes longs sont imprimés, non plus sous une forme classique, mais sous la forme de l'impression qui est seconde par rapport à la réception électronique. On le voit dans l'économie domestique: Iorsqu'on vous envoie un « document attaché », le coût de l'impression relève du récepteur et non plus del'expéditeur. À une échelle beaucoup plus grande, c'est la fameuse phrase de Bill Gates: "Q uand je veux vraiment lire un livre, je l'impri- 
me. » Ce qui veut dire que l'impression est quasiment considérée comme une partie du processus de la transmission électronique, jusqu'au moment où des lecteurs - même face à un ouvrage de longue taille, dans une lecture continue -, n'auront plus besoin de passer à cette impression, parce qu'ils pourront le porter sur eux, dans des portables qui seraient vraiment plus portables que ce que nous nous appelons portables, et qui auraient la forme du «e book ». L'idée des échelles de coûts est donc finalement assez intéressante, puisqu'on a vu sopérer un basculement des coûts, de ceux qui les supportaient jusqu'à maintenant sur de nouveaux payeurs. Pour illustrer ce que vous dites, il me parait qu'il y a deux exemples qui seraient très intéressants, et qui ont été discutés récemment à une journée de la M aison des écrivains: ce sont les écrivains qui, à la fois, ont un site web sur lequel ils font circuler en accès gratuit un certain nombre de textes, et qui publient, soit avec des éditeurs classiques, soit avec des éditeurs électroniques. Cela suppose dans les deux cas qu'il y ait un paiement du lecteur, soit qu'il achète le livre, soit qu'il doive payer la réception du texte électro- nique. C ela me semble un exemple très intéressant parce que leur position est assez différente en fonction de ce qu'ils distribuent de leur écriture dans l'une et l'autre forme. Pour certains, I'œuvre elle-même appartient toujours au monde de l'édition, et ce qui est donné en libre accès c'est tout ce qui ressort des entours de l'œurre: brouillons, esquisses, informations, textes plus personnels, etc. Pour d'autres, au contraire, c'est une manière d'entrer dans une gratuité de la communication esthétique, ce qui renverrait sans doute aussi à Bourdieu, à une sociologie des positions des auteurs, parce qu'il est facile d'être gratuit lorsqu'on a les moyens de l'être, alors que pour certains auteurs qui, dans la continuité du copyright du XVIII siècle, essayent de vivre de leur plume, la gratuité de la communication esthétique est contradictoire avec leur propre existence sociale.

On ne peut pas discuter simplement en des termes abstraits de ce qui serait le souhait désirable d'une communication textuelle libre et gratuite car elle renvoie aussi à une sociologie des positions. La position des auteurs de sciences humaines et sociales, qui sont très rarement des professionnels de 
l'écriture, mais plutôt des gens qui ressemblent à des bénéficiés de l'Ancien Régime - ils ont un emploi, un office, et peuvent avoir des droits d'auteur, mais leur existence sociale n'est pas mise en jeu par la vente ou la circulation monnayée de leurs textes -, est différente de celle de beaucoup d'écrivains de fiction, dont c'est l'existence même comme auteur qui vit de sa plume qui est en question. On peut le voir à toutes les échelles: à l'échelle de ceux qui en vivent mal, mais qui vivent quand même de l'écriture et qui se trouvent confrontés à cette tension entre communication gratuite et droits économiques sur l'œuvre; à l'échelle des auteurs de best-sellers - et vous avez vu comment Stephen King, après sa première expérience, avait essayé une seconde expérience, c'est-à-dire de se faire éditeur lui-même, de ne plus passer par un éditeur qui était en même temps un éditeur électronique, comme Schuster, mais d'essayer d'être son propre éditeur avec la difficulté qui est née de la lourdeur des modes de paiement. Ça n'a pas été un énorme succès, mais on voit que la question se pose à toutes les échelles du champ littéraire. Cette tension existe aussi pour les édi- teurs, puisque I'on constate que lorsque les éditeurs ont un site électronique - et donc un site en accès gratuit par rapport à la vente, soit en ligne, soit en librairie - , ce sont seulement des informations sur les titres, sur les auteurs qui sont publiés et pas les textes eux-mêmes, ce qui serait contradictoire avec l'activité éditoriale.

Vous avez raison de souligner, un peu à la Walter Benjamin, qu'il n'y a pas de fatalité dans les objets techniques: ce sont des possibilités qui sont offertes et qui sont finalement exploitées ou non. En même temps, il me semble que ce monde du partage des textes dans des formes différentes est un monde auquel arrivent les éditeurs, les auteurs, les fabricants de machines, confrontés à cette situation si complexe qui est à la fois économique, intellectuelle, esthé tique et éthique et qui pose la question du rapport entre le droit et le marchéd'un côté et, del'autre la communauté et l'échange. 


\section{Les métamorphoses du livre}

Après avoir étudié les conditions de production et de diffusion traditionnelles des livres lors des « Rendez-vous de l'édition \%, la Bibliothéque propose, en prolongement de ce cycle, les rencontres a Les rendez-vous de l'édition: le livre ot lo numérique $\%$, afin do donnor au public dos informations et des repères pour comprendre les évolutions en cours. En effet, les technologies numériques permettent de développer ou de renouveler de nombreux produits et services: édition en ligne, livre électronique, librairies virtuelles, impression à la demande, et de nouveaux besoins et comportements face à l'accts aux contenus émergent. Comment les acteurs de la chaîne du livme s'y adaptent-ils et quelle est l'évolution du rôle des uns et des autres : auteurs, éditeurs, distributeurs, libraires, bibliothecaires? Quelles mutations des pratiques de lecture peut-on observer? Le livre, support essentiel de la culture écrite pendant des siècles, se trouve aujourd hui fortement concurrencé par les technologies numériques. Le regard d'un historien, Roger Chartier, permet de comprendre l'originalité de cette troisième révotution du livre qui est tout à la fois révolution de la technique de production ot de reproduction des textes, róvolution du support de l'écrit et révolution des pratiques de lecture. Ces nouvelles modalités de production, de transmission et d'appropriation culturelles suscitent des tensions majeures qui rendent nécessaires une réflexion historique, sociologique et politique impliquant chacun d'entre nous.

D. Epilesntre Pormpidou. 2001.

190N 2-84246-069-3

Horts commerce 